\title{
Correction to: Decision Science for Future Earth: A Conceptual Framework
}

Tetsukazu Yahara, Wataru Tanaka, Yukako Inoue, Jounghun Lee, Kun Qian, Firouzeh Javadi, Nariaki Onda, Fumihiko Yokota, Kumi Eguchi, Mariko Nishikitani, Kimiyo Kikuchi, Nobuyoshi Kawasaki, Yukyong Jeong, Jun'ichiro Ide, Tetsuji Ota, Takahiro Fujiwara, Tadatsugu Hosoya, Yuichi Kano, Megumi Sugimoto, Ashir Ahmed, Yukihiro Shimatani, Shota Tokunaga, Ai Nagahama, Michikazu Hiramatsu, and

Takahiro Murakami

\section{Correction to:}

Chapter 1 in: T. Yahara (ed.), Decision Science for Future

Earth, https://doi.org/10.1007/978-981-15-8632-3_1

This chapter was inadvertently published with an incorrect forename of an author. The correct forename of the 12th author is Nobuyoshi to read as 'Nobuyoshi Kawasaki'.

The author name has been updated with this erratum. 